\title{
LA REGIÓN METROPOLITANA DE BARCELONA. DINÁMICAS TERRITORIALES RECIENTES'
}

\author{
Carme Miralles-Guasch y Antoni F. Tulla Pujol \\ Departamento de Geografía. Universitat Autònoma de Barcelona
}

\section{RESUMEN}

Las dinámicas sociales y económicas urbanas se dan en los espacios metropolitanos, por lo que las áreas metropolitanas se han convertido en las unidades territoriales de análisis. De ahí que sean observatorios privilegiados desde donde estudiar la realidad urbana actual. Aquí se presentan las dinámicas territoriales recientes de la región metropolitana desde la perspectiva de la redistribución poblacional y de las actividades económicas y, también, de las tipologías de viviendas construidas. Estas variables introducen subámbitos metropolitanos y, a través de ellos, se analiza el mapa administrativo y los modelos de movilidad.

Palabras clave: ciudad, área metropolitana, movilidad, Barcelona.

\section{ABSTRACT}

Social and economic dynamics appear in metropolitan territories, and as such metropolitan areas have become territorial units of analysis. Hence they have become privileged observatories for the study of current urban reality. Here recent territorial dynamics of the metropolitan region are presented from the perspective of population redistribution and economic activities as well typologies of built housing. These variables introduce metropolitan subareas and through them administrative maps and mobility models are analyzed.

Key words: city, metropolitan area, mobility, Barcelona.

Fecha de recepción: septiembre 2010.

Fecha de aceptación: febrero 2012.

1 Este artículo ha sido financiado por el Proyecto CSO2010-18022 (subprograma geog): La perspectiva territorial, social y medioambiental en las investigaciones sobre movilidad y transporte. Un análisis desde la Geografía. 


\section{INTRODUCCIÓN}

El estudio de los fenómenos urbanos a finales del siglo XX y principios del siglo XXI tiene un fuerte componente metropolitano. Las grandes transformaciones socioeconómicas del mundo global actual no se dan en núcleos aislados, sino en territorios definidos por interrelaciones entre ciudades diversas en tamaños y perfiles. El fenómeno metropolitano se ha convertido en la expresión espacial de la globalización (Belil, 2003) y, aunque parezca paradójico, a partir de los años noventa, precisamente después de las aboliciones del Consejo del Gran Londres, del Rijnmond de Rotterdam y de la Corporación Metropolitana de Barcelona, se iniciaron nuevos debates sobre el sistema urbano europeo y se crearon o redefinieron instituciones metropolitanas (Londres, Bolonia, Stuttgart, Hannover y Copenhague, por ejemplo). Dichas estrategias eran reacciones políticas ante las disfunciones administrativo-territoriales y las crisis de décadas anteriores (Brenner, 2006). Este resurgimiento se enmarca en la aparición del regionalismo metropolitano en Europa occidental, como expresión de procesos subnacionales definidos como neorregionalismo (Amin, 1999; Lovering 1999; Macleod 2001).

De ahí que, en palabras de Méndez (2009), los espacios metropolitanos se hayan convertido en observatorios privilegiados desde donde analizar la realidad urbana y social actual. No en vano, Sassen y Castells, en muchas de sus obras abordan desde distintas perspectivas metodológicas el papel crucial de las grandes metrópolis en el proceso de crecimiento económico (Trullén, 2003). En ese proceso, el territorio se percibe como un factor esencial en el crecimiento económico de las últimas décadas (Florida, 2008; Salom, 2010).

Muchas de las áreas metropolitanas, y Barcelona no es una excepción, se han formado alrededor de ciudades centrales maduras, con alta densidad de población y con una jerarquía urbana muy marcada. Sin embargo, hace ya algunas décadas que el crecimiento periférico de los sistemas metropolitanos ha dejado de tener formas y funciones homogéneas y subalternas. Fenómeno pronosticado ya, en los años sesenta y setenta, por los trabajos pioneros sobre suburbanización de Berry, Hall \& Hay y Van den Berg y que ahora define a casi todas las metrópolis del mundo occidental (Marmolejo y Roca, 2008). En diversas realidades metropolitanas americanas y europeas se asiste a una sustitución de la antigua estructura monocéntrica y jerárquica por otra más policéntrica y reticular (Filion et ál. 1999; Ferrao, 2004; Méndez, 2009). Metrópolis policéntricas donde, desde distintas disciplinas de las ciencias sociales, se están investigando las dinámicas territoriales que las optimizan, según pautas de redistribución de la población, de la ocupación del territorio y de los modelos de movilidad. Investigaciones que buscan pautas y estrategias territoriales entre la multiplicación, la diversificación y la especialidad de las distintas centralidades y la inclusión de áreas de influencia cada vez más alejadas y sin continuidad urbana en el espacio, aunque fuertemente interconectadas (Belil, 2003), con un creciente protagonismo de sus periferias, convertidas en espacios dinámicos y diversos (Valenzuela Rubio y Salom Carrasco, 2008; Méndez, 2007), aunque les falte relato territorial y carezcan, en muchos casos, de nodos de centralidad (Muñoz, 2008).

En consecuencia, resulta imprescindible adoptar un cambio de perspectiva escalar en el análisis territorial entre los espacios suburbanos metropolitanos y las amplias regiones urbanas periféricas (Salom, 2010), acompañadas por un replanteamiento teórico que substituya la tradicional interpretación del territorio de relaciones jerárquicas entre núcleos, por otra 
de carácter horizontal. Aunque todo ello, como indica Feria (2004), dificulte enormemente homogeneizar fuentes de información, encontrar el ámbito adecuado de análisis e incluso barajar conceptos transversales que permitan estudios comparados.

Algunas variables se perciben como fundamentales para entender la situación descrita. Una de ellas es la relocalización de las actividades económicas, tanto de las tradicionales, referidas al sector industrial, como de otras relativamente nuevas y con un protagonismo creciente, relacionadas con el conocimiento (Caravaca y Méndez, 2003; Méndez, 2007). Otras, enlazadas con la primera, se centran en los flujos de personas y en los consecuentes modelos de movilidad que esas metrópolis generan (Miralles-Guasch, 2002). Tanto una como las otras inciden en la vida cotidiana organizada dentro del tejido metropolitano en múltiples polos, a menudo, alejados entre sí (Ferrao, 2004) y en la reubicación de la población de las metrópolis en núcleos medianos y pequeños (Pujadas, 2009). Todo ello en coherencia con los incrementos de las accesibilidades y las reformulaciones de las distancias y los tiempos de la movilidad (Oyón, 1999; García, 2008).

Las alteraciones en las dinámicas territoriales reflejan cambios en la base económica de las ciudades que, a menudo, se traducen en una reubicación territorial. Si en un principio las actividades se localizaban dentro de las tramas urbanas tradicionales de los grandes núcleos urbanos de los territorios metropolitanos, desde hace algunos años, se van ubicando en espacios periféricos, más o menos aislados, y en núcleos medianos y pequeños, con buenas comunicaciones con el centro metropolitano (Crevoisier y Camagni, 2002; Muñiz y García López, 2010), proceso que se intensifica en las ciudades maduras y en las áreas metropolitanas de mayor rango (Sassen, 2003).

Sin embargo, la evolución de la localización de la actividad económica en las metrópolis no ha tenido un recorrido uniforme, todo lo contrario. A estos procesos de desconcentración se le suman en paralelo dinámicas de concentración de nuevas actividades, las llamadas funciones centrales (Caravaca y Méndez, 2003) o actividades del conocimiento y la información, que forman aglomeraciones localizadas en el centro de las principales áreas metropolitanas (Cuadrado y Del Río, 1993).

Dos elementos influyen enormemente en estos procesos. Uno es la estructura del mapa administrativo que, o bien permite la gestión integrada de un territorio metropolitano cada vez más interdependiente, o bien, por el contrario, lo hace imposible debido a la multiplicidad de Gobiernos locales y agentes privados existentes. El otro elemento son las grandes infraestructuras relacionadas directamente con los cambios de modelo urbano descritos (García, 2001). Véase como ejemplo la construcción del puente de Oresund entre Dinamarca y Suecia, que ha permitido la consolidación de un área urbana de más de tres millones de habitantes alrededor de las ciudades de Calmo y Copenhague, o la línea de alta velocidad que une las capitales de Londres, París y Bruselas, y que influye en la transformación y potenciación de la región metropolitana transfronteriza de Lille (Belil, 2003). También los trabajos de Bellet (Bellet et ál. 2010) de las áreas metropolitanas de España, en relación con la puesta en marcha del tren de alta velocidad, verifican la ampliación de ciertas áreas metropolitanas españolas, con la inclusión de núcleos situados a una hora de viaje de la capital.

Estos efectos territoriales de las infraestructuras se traducen en modelos de movilidad característicos de los ámbitos metropolitanos (García, 2008). De hecho, los análisis de la movilidad siempre han estado unidos a los estudios de las dinámicas metropolitanas. Desde 
distintas disciplinas sociales, utilizando diversas variables analíticas, se han identificado modelos territoriales con la movilidad. Tres grandes líneas de investigación han desarrollado esta relación: una de ellas, articulada por los economistas urbanos, donde el precio del transporte es el elemento central de la redistribución de la población y de las actividades económicas en los ámbitos metropolitanos ${ }^{2}$; otra, visualizada desde la geografía, es la que identifica unidades territoriales con mercados de trabajo a partir de los orígenes y los destinos de los desplazamientos intermunicipales por motivos laborales, como una aproximación cuantitativa a la realidad metropolitana ${ }^{3}$; por último, otros trabajos han constatado empíricamente las relaciones entre las pautas en la distribución de los usos, las densidades, la morfología o el propio diseño urbano con las demandas de movilidad. En ellos se señalan los cambios en los procesos territoriales como una de las causas de los incrementos y las diversidades de la movilidad actual (Priemus et ál. 2001; Stover y Koepke, 2002; Camagni et ál. 2002; Rodríguez y Joo, 2004; Mella y López 2006), o hacen hincapié en la relación entre los modelos de movilidad y la realidad urbana a partir de las características de los desplazamientos, en lo que se refiere al transporte utilizado, al tiempo y al motivo (Orfueil y Salomon, 1993; Giulano y Small, 1999; Gutiérrez y García 2005, 2007).

\section{DINÁMICAS TERRITORIALES}

La región metropolitana de Barcelona (RMB), como otros espacios urbanos maduros del mundo occidental, en los últimos años está sumida en una transformación de modelo territorial y socioeconómico. Evolución que subraya, tal y como se ha indicado en la introducción, el papel de las periferias metropolitanas y acentúa en la RMB su carácter de metrópoli policéntrica (Nel.lo, 2001) donde los núcleos de rango menor tienen cada vez más importancia y más presencia.

Sin embargo, en la RMB el modelo urbano es dual (Miralles-Guasch, 1998; Marmolejo y Roca, 2008), si bien las periferias ganan protagonismo, cuantificado en términos de población y actividades económicas, el centro también actúa como una ciudad capital intensa en actividad económica y muy activa en términos culturales y sociales. Un modelo dual al que un mapa administrativo fragmentado no le es ajeno, con una morfología urbana que se debate entre la compacidad y la dispersión, unas densidades de población desiguales y unas actividades económicas que se empiezan a relacionar con los territorios y las economías del conocimiento.

\section{Redistribución de la población entre los ámbitos metropolitanos}

La distribución y la dinámica demográfica de la RMB tienen una evolución similar a la de otras metrópolis maduras del mundo occidental. La disposición de la población en la RMB define tres ámbitos territoriales, equilibrados en población pero con características poblacionales y socioeconómicas distintas. En primer lugar, el municipio de Barcelona, con

2 En España destacan los trabajos de Anna Matas, Ginés de Rus y Germà Bel.

3 En España destacan los trabajos de Josep Serra, Pilar Riera, Margarita Castañer, José M.Feria y Juan Susino, Julia Salom y Antonio Casado. 
1.615.908 habitantes y unas densidades cercanas a los $16.000 \mathrm{~h} / \mathrm{km}^{2}$. Después, la primera corona metropolitana, o aglomeración central, con una estructura urbana compacta alrededor de Barcelona e integrada por 26 municipios; su población es de 1.570.553 habitantes y la densidad de población es de unos 2.900 habitantes $/ \mathrm{km}^{2}$. Por último, el tercer ámbito territorial corresponde a la parte más externa de la región, o segunda corona metropolitana, integrada por ciudades medianas, que actúan de capitales intermedias, de núcleos pequeños y de zonas rurales, con una población de 1.742.391 habitantes y una densidad media de unos 668 habitantes $/ \mathrm{km}^{2}$ (Figura 1).

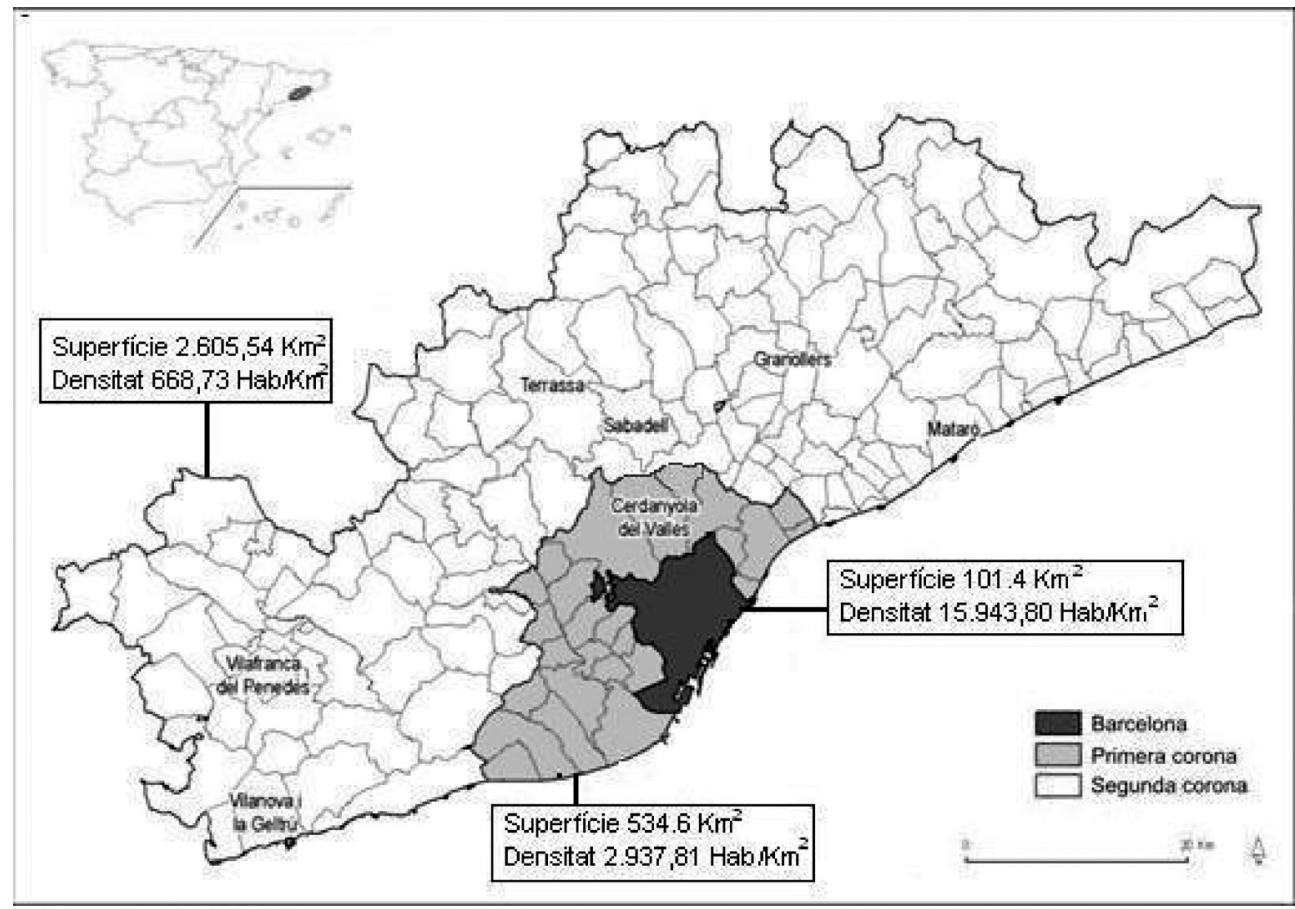

Fuente: Elaboración propia a partir de IDESCAT y web Área Metropolitana de Barcelona.

Estas distintas densidades entre los ámbitos metropolitanos barceloneses indican dos formas de urbanizar y de ocupar el territorio. En el centro de la metrópolis, Barcelona y su continuo urbano, existe una urbanización compacta en su forma y compleja en sus funciones, derivada de una apuesta técnico-política por una ciudad densa y continua de los espacios urbanos. Y, además, con una distribución de las funciones urbanas homogénea y mixta, por lo que a lo largo de toda la ciudad no existen espacios destinados a una sola función, ya sea comercial, residencial o industrial. Por el contrario, el modelo de ocupación de la periferia metropolitana (Muñoz, 2006), más en la segunda corona que en la primera, y exceptuando los núcleos históricos de sus ciudades medias (señaladas en la figura 1), se 
caracteriza por el funcionalismo zonal, la discontinuidad de los tejidos urbanos y las bajas densidades.

Distribución de la población que, en las últimas décadas, se ha encaminado hacia la dispersión en el territorio, desde las ciudades más pobladas y de mayor densidad hacia núcleos pequeños y zonas de baja densidad (Tabla 1) (Pujadas, 2009). Desde los años ochenta, en Barcelona y en otras ciudades medias del entorno metropolitano se han producido pérdidas de población debidas a migraciones residenciales. El resultado ha sido la disminución del peso demográfico de Barcelona en relación con el resto del territorio de la RMB, que ha pasado de ser el $41 \%$ en 1981, al $33 \%$ en 2008. En las coronas metropolitanas, y especialmente en la segunda, en su parte más periférica, se ha experimentado un proceso inverso con el aumento de la población por saldos migratorios positivos. Entre 1981 y 2008 la población de la segunda corona metropolitana pasó de poco más de un millón de personas a más de 1.700 .000 , lo que significa un aumento del $52 \%$. El crecimiento de la primera corona ha sido mucho más discreto, con un aumento de la población de apenas 180.000 personas, lo que significa un incremento del $14 \%$. Una dinámica de población que indica la creciente importancia de las periferias metropolitanas en los contextos urbanos actuales (Méndez, 2009).

Tabla 1

EVOLUCIÓN DE LA POBLACIÓN EN LOS DISTINTOS ÁMBITOS DE LA REGIÓN METROPOLITANA DE BARCELONA

\begin{tabular}{|l|c|c|c|c|}
\hline & \multicolumn{2}{|c|}{ Población 1981 } & \multicolumn{2}{c|}{ Población 2008 } \\
\hline & Habitantes & \% & Habitantes & $\%$ \\
\hline Barcelona & 1.752 .627 & $41 \%$ & 1.615 .908 & $33 \%$ \\
\hline Primera corona & 1.392 .386 & $33 \%$ & 1.570 .553 & $32 \%$ \\
\hline Segunda corona & 1.093 .863 & $26 \%$ & 1.742 .391 & $35 \%$ \\
\hline Total RMB & 4.238 .876 & $100 \%$ & 4.928 .852 & $100 \%$ \\
\hline
\end{tabular}

Fuente: Área Metropolitana de Barcelona.

Todo ello en paralelo a la importancia creciente de los tejidos residenciales de baja densidad, que no pueden ser tratados como un fenómeno minoritario en la RMB, pues grandes zonas del territorio se caracterizan por esta tipología de vivienda. En el año 2000 se contabilizaban $6.089,2$ ha de suelo urbano residencial extensivo y de baja densidad, lo que representa un 40 $\%$ del total del suelo residencial (MMAM, 2003). Actualmente, la producción de vivienda está generando unas tipologías de residencias que incrementan la presencia en la RMB de esos tejidos. Así, entre 1987 y 2001 se construyeron en la RMB 330.511 viviendas, de las que más de 205.000, es decir el $65 \%$ correspondían a tipologías de baja densidad - viviendas aisladas, adosadas o unifamiliares -, (Muñoz, 2005). El mayor número absoluto de viviendas de este tipo, en concreto 206.878, se construyeron en la segunda corona metropolitana.

El resultado de esta dinámica de dispersión se puede observar en la figura 2, en la que se expresan los porcentajes de vivienda en alta y baja densidad en los tres ámbitos metropolitanos mencionados. Las tipologías de vivienda de baja densidad incrementan su presencia a medida que nos alejamos del centro de la metrópolis, y llegan casi al 40\% del total del parque de viviendas en la segunda corona metropolitana (Miralles-Guasch y Donat, 2007). 
TIPOLOGÍA DE VIVIENDAS EN LA RMB 2006

Barcelona

Fuente: ECVHP, 2006.

\section{Redistribución de las actividades económicas entre los ámbitos metropolitanos}

Con relación a la distribución y evolución de las actividades económicas, en los últimos años se desprenden unas conclusiones similares a las de la población, existe un proceso de desplazamiento de las actividades económicas hacia zonas externas de los municipios, en busca de mejores condiciones y accesibilidades, aunque Barcelona y los municipios del núcleo central metropolitano no dejan de tener un gran dinamismo económico. Esta tendencia se inicia con las actividades industriales y, más recientemente, se le suman el sector terciario, el comercio e incluso los centros educativos y sanitarios. Sin embargo, tal como apuntan Marmolejo y Roca (2008), existe también una pauta de localización compacta, recentralizante y concentrada de las actividades inmateriales relacionadas con la gestión de la información y el conocimiento que acentúa el modelo urbano dual explicitado al principio.

La distribución y la evolución de los afiliados en la seguridad social dan cuenta de este proceso de descentralización de la actividad económica. Entre 1996 y 2008, en el conjunto de la RMB, el número de afiliados a la seguridad social se incrementó el 31\%, aunque con un crecimiento desigual según los ámbitos. En la ciudad de Barcelona el aumento fue del $27 \%$, sin embargo, es en la segunda corona metropolitana donde se llegó al 39 \% (Tabla 2).

Tabla 2

DISTRIBUCIÓN DE LOS PUESTOS DE TRABAJO EN LA REGIÓN METROPOLITANA DE BARCELONA

\begin{tabular}{|l|c|c|c|}
\hline \multirow{2}{*}{\multicolumn{1}{|c|}{ Ámbito }} & \multicolumn{2}{c|}{ Puestos de Trabajo } & Incremento \\
\cline { 2 - 4 } & $\mathbf{1 9 9 6}$ & $\mathbf{2 0 0 8}$ & $\mathbf{1 9 9 6 - 2 0 0 8}$ \\
\hline Barcelona & 787.507 & 1.078 .820 & $27 \%$ \\
\hline Primera corona & 387.441 & 563.264 & $31 \%$ \\
\hline Segunda corona & 422.897 & 689.970 & $39 \%$ \\
\hline Total RMB & 1.599 .841 & 2.332 .054 & $31 \%$ \\
\hline
\end{tabular}

Fuente: Mancomunidad de Municipios del Área Metropolitana de Barcelona, 2006 y Ayuntamiento de Barcelona; http://www.bcn.es/publicacions/b_economia/be68.pdf 
El proceso de difusión de las actividades por el territorio se ha producido en paralelo a la proliferación de polígonos industriales. En la RMB se concentran un total de 712 polígonos de actividad que suman 15.653 ha y representan casi el $50 \%$ del total catalán. Su localización, tal como se observa en la figura 3 se concentra en las partes más alejadas de la RMB, siguiendo las trazas de las redes viarias de alta capacidad. Muchos de estos polígonos están ubicados en zonas externas de la ciudad y aislados de las partes urbanas consolidadas (Miralles-Guasch y Donat, 2007).

Sin embargo, a esos procesos de difusión se le contraponen dinámicas territoriales derivadas de las actividades de la información y el conocimiento, con menores niveles de difusión. Estas actividades se concentran en el centro de la metrópolis, en este caso, en ciertos barrios de la ciudad de Barcelona (Trullén et ál. 2002; Capel, 2005). En palabras de Marmolejo y Roca (2008): «en la medida que incrementa la distancia desde el centro hacía la periferia aparece un gradiente descendiente de pérdida de especialización en estas actividades». En la RMB las actividades industriales se están redistribuyendo, impulsadas por los efectos de la congestión y la densidad de los centros, sin embargo, este fenómeno se alterna con los efectos de las economías de aglomeración ligados a sectores de alto valor añadido (Muñiz y García López, 2010).

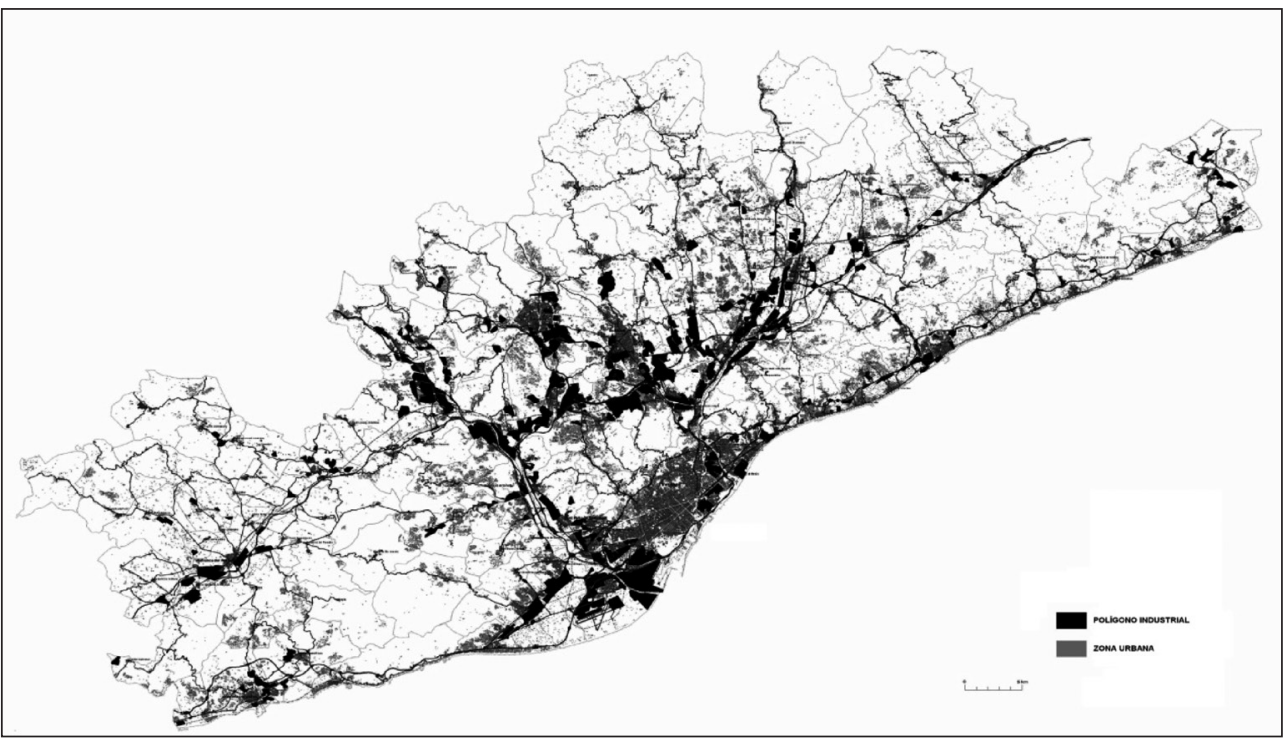

Fuente: Elaboración propia a partir de Miralles y Donat, 2007.

\section{Una región metropolitana con un mapa administrativo fragmentado}

Ya a mediados del siglo XX, el entorno metropolitano barcelonés requería de una visión territorial a una escala más amplia que la estrictamente municipal. Con este propósito se 
intentó una planificación supramunicipal (a partir de dos planes urbanísticos), fracasada en su momento, que fue el embrión de los planes metropolitanos posteriores.

El plan de 1953, fue el primer plan urbanístico supramunicipal que incluía Barcelona y los 26 municipios de su alrededor, con un total de $456 \mathrm{Km}^{2}$, denominado Plan de Ordenación de Barcelona y su zona de influencia. Un ámbito donde, en los años setenta, se ubicaría el Plan General Metropolitano, un plan urbanístico supramunicipal aún hoy vigente. Este territorio también se organizó como la Corporación Metropolitana de Barcelona que, a nivel estadístico, se denomina primera corona metropolitana (Figura 1).

En la década de los sesenta se presentó el segundo intento con el Plan Director del Área Metropolitana de Barcelona, más amplio que el anterior, con la inclusión de las siete comarcas alrededor de Barcelona y sus 166 municipios. Es el ámbito de lo que hoy se conoce como región metropolitana de Barcelona (RMB) y es donde se articulan los últimos intentos de planificación y ordenación metropolitana conjunta (Figura 1).

La falta de voluntad política, de instrumentos técnicos y de normativas legislativas para desarrollar un proyecto supramunicipal y los frustrados inicios de la planificación metropolitana malograron todos los intentos, a excepción del Plan General Metropolitano de 1976 aún hoy vigente. Buen ejemplo de ello fue la supresión en 1987 de la Corporación Metropolitana de Barcelona - el único ente de acción conjunta metropolitana que ha existido- y la creación de tres mancomunidades de municipios, con el objetivo de gestionar los residuos, el agua y los transportes, sin que ninguna de ellas tuviera una visión de conjunto. Cada una incluye municipios distintos, que en su totalidad suman 36 Administraciones locales alrededor de Barcelona. En este ámbito es en el que recientemente se ha organizado de nuevo el área metropolitana.

Este cambio de rumbo metropolitano de finales de los años ochenta también significó el inicio del tercer intento de una planificación supramunicipal, con el Plan Territorial. El objetivo era abordar la ordenación del espacio metropolitano en un ámbito más real, por lo que se adoptó el perímetro del antiguo Plan Director de los años sesenta. Y aunque se terminó en 1998, nunca se aprobó, no obstante consolidó lo que hoy se conoce como la región metropolitana de Barcelona (RMB), ámbito territorial utilizado en los trabajos de planificación territorial y de infraestructuras recientes (Figura 1).

En toda la RMB las únicas unidades administrativas legalmente constituidas son los municipios, que con un total de 166, fragmentan enormemente el mapa metropolitano y dificultan cualquier intento de planeamiento a escala metropolitana. Y también las comarcas, siete en total, que al ser unidades muy pequeñas, sin muchas competencias reales, no permiten una gestión supramunicipal adecuada. Y en el núcleo central de la metrópolis ha aparecido el área metropolitana, sin eliminar ningún límite administrativo, por lo que en esta zona existen cinco niveles administrativos distintos.

El fracaso de todo este proceso, que caracteriza el planeamiento supramunicipal de la RMB en la segunda mitad del siglo XX, confirma la validez de la idea de mediados de los noventa de Caravaca y Feria (1994) referente a la debilidad y a la fragmentación de las zonas metropolitanas españolas. Y, en particular, para Barcelona esta fragmentación da lugar a una planificación a escala municipal en la segunda corona que se expresa en crecimientos dispersos y muy poco sostenibles (Carreras, 2002).

Sin embargo, esta tendencia puede cambiar, ya que a mediados del año 2010 el Parlamento catalán aprobó el Plan Metropolitano de Barcelona (PTMB) (Generalitat de Catalunya, 
PTOP, 2010) para un ámbito de 7 comarcas y 166 municipios. Documento de planeamiento territorial que obliga, a las Administraciones competentes, a establecer unos criterios de planeamiento, de intervención y de control unificado para esta área.

\section{LA MOVILIDAD EN LA REGIÓN METROPOLITANA DE BARCELONA}

Los desplazamientos están directamente relacionados con la distribución de las actividades económicas sobre el territorio. En esta dialéctica, los tiempos, los motivos y los medios de transporte de un ámbito metropolitano derivan de variables que dependen de las distribuciones espaciales de las actividades y de los tipos de urbanización.

\section{Las características de la movilidad en la región metropolitana de Barcelona}

Entre 2004 y 2006 el número de desplazamientos, según las encuestas de movilidad ${ }^{4}$ en la RMB, aumentaron un 20\%, y en 2008 se estabilizaron alrededor de unos 14 millones de movimientos diarios. Con un ratio que oscila entre los 3,47 y los 3,64 desplazamientos por persona y día. También se observa un aumento de la longitud de desplazamientos por motivos de trabajo y estudio (EMO, 2001) ${ }^{5}$, entre 1981-2000 la distancia media ha pasado de 9,6 a 12,9 Km.

Por lo que se refiere a los modos de transporte utilizados, de los 101,2 millones de desplazamientos semanales hechos por los residentes de la región metropolitana de Barcelona, 45,8 millones $(45,2 \%)$ se realizan en modos no motorizados; 38,2 millones $(37,8 \%)$ en transporte privado y 17,2 millones $(17,0 \%)$ en transporte público (Figura 4). En día laborable el uso del transporte público es más elevado que en sábados y festivos (el 18,6\% y el 11,1\%, respectivamente), tendencia que se invierte en el uso del transporte privado (35,8\% y 44,7\%, respectivamente).

Figura 4

DISTRIBUCIÓN MODAL DE LOS DESPLAZAMIENTOS SEMANALES EN LA REGIÓN METROPOLITANA DE BARCELONA. EMQ 2006

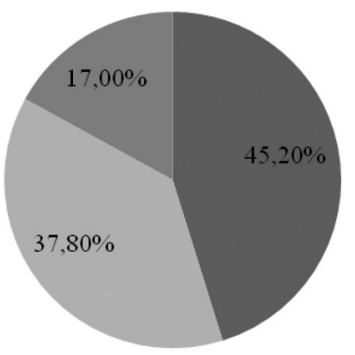

\begin{tabular}{|l|l|}
\hline Vehiculo privado & \\
\hline No motorizado (a pie + bicicleta) & \\
\hline Transporte público & \\
\hline
\end{tabular}

Fuente: ATM, PTOP, EMQ 2006.

4 La información sobre la movilidad de las personas en la región metropolitana de Barcelona se basa en un sistema de encuestas periódicas. Anualmente se realiza la encuesta de movilidad en día laborable (EMEF) y, con periodicidad quinquenal, la encuesta de movilidad cotidiana (EMQ).

5 Hasta 2001 se realizaba la encuesta de movilidad obligada que, a pesar de su nombre, no consistía en una encuesta sino en la recopilación de información a partir del censo. Permitía un estudio exhaustivo de la población con gran desagregación espacial, aunque sólo recogía información sobre el primer desplazamiento por motivo de trabajo o estudio. 
En la RMB los desplazamientos no motorizados, y especialmente el ir andando, son una parte fundamental de la movilidad metropolitana, pues la utilización de la bicicleta no llega al $2 \%$. En transporte público, los modos más urbanos como el metro y el autobús son los más usados, con el 33,4\% y el 28,1\% del total de recorridos hechos en este medio de transporte en días laborables. En el transporte privado, el coche es el más utilizado con una cuota del $86,9 \%$ en días laborables y el $94,0 \%$ en fines de semana, con una ocupación media que nunca supera los dos individuos por vehículo.

Los dos modelos urbanos mencionados anteriormente entre el centro metropolitano y sus periferias se traducen en dos modelos de movilidad distintos, según los usos de los diversos modos de transporte. Las altas densidades urbanas y una mayor oferta de transporte público en Barcelona y su continuo urbano ${ }^{6}$ determinan que, en este ámbito, sea donde más se utilice este transporte, con cuotas de alrededor del $30 \%$ en día laborable y del $20 \%$ en día festivo. Este hecho, junto con un alto porcentaje de desplazamientos a pie, que representan casi la mitad de todos los movimientos, influye en un menor uso del transporte privado en esta área central de la RMB. A medida que nos alejamos del centro de la metrópolis emerge todo lo contrario, el peso del transporte privado es cada vez mayor en menoscabo del uso del transporte público (Figura 5).

Figura 5

MEDIOS DE TRANSPORTE EN LA REGIÓN METROPOLITANA DE BARCELONA EN DÍA LABORABLE, 2006

\begin{tabular}{|c|c|c|}
\hline Barcelona & Primera Corona & Segunda Corona \\
\hline Transporte público & $\square$ Medios no motorizados $\square$ Vehiclulo privado \\
\hline $23,1 \%$ &
\end{tabular}

Fuente: ATM, PTOP, EMQ 2006.

Por lo que se refiere a los motivos ${ }^{7}$ de desplazamiento (Figura 6) de los residentes en la región metropolitana de Barcelona, alrededor del $34 \%$ son personales, un $20 \%$ ocupacionales y el 45,5\% se concentran en los retornos al lugar de residencia. Los motivos de desplazamiento son distintos según el día de la semana. Las principales diferencias se producen en los motivos ocupacionales, que en los fines de semana se reducen al 3,7\% del total, en beneficio de la movilidad personal que llega a ocupar hasta el 50\% de la cuota. A nivel terri-

6 El continuo urbano tiene unos límites parecidos a la primera corona metropolitana.

7 La Encuesta de Movilidad Cotidiana 2006 diferencia tres grupos de motivos: el ocupacional, el personal y los regresos al lugar de residencia. En los primeros se agrupan los motivos laborales y de estudios. Entre los personales se encuentran las compras, el ocio, las visitas, los paseos, etc. En total, en dicha encuesta se hallan 14 motivos distintos que originan los desplazamientos. 
torial destaca el peso que las razones personales tienen en la ciudad de Barcelona, tanto en día laborable como en festivo. La estructura de edades de la población puede explicar esta diferencia territorial.

Figura 6

DISTRIBUCIÓN DE DESPLAZAMIENTOS SEGÚN MOTIVO EN DÍA LABORABLE. 2006

\begin{tabular}{|l|l|l|l|}
\hline & & & \\
\hline
\end{tabular}

Fuente: ATM, PTOP, EMQ 2006.

Puede observarse, también, una correlación entre los modos de transporte y los motivos. El 46,8\% de la movilidad por trabajo y estudio se realiza en vehículo privado. Este predominio deriva de las actividades y del proceso de dispersión de la población que se ha producido en los últimos años, dispersión que obliga a recorrer grandes distancias para acceder al puesto de trabajo y en las que el vehículo privado resulta imprescindible, especialmente en los polígonos industriales localizados en los espacios suburbanos (Cebollada, 2006). Por el contrario, la movilidad a pie predomina cuando los motivos del desplazamiento son personales (compras, ocio, visitas...), con un porcentaje de casi el 60\% (Figura7).

Figura 7

MOTIVOS Y MEDIOS DE TRANSPORTE EN DÍA LABORABLE. EMQ 2006

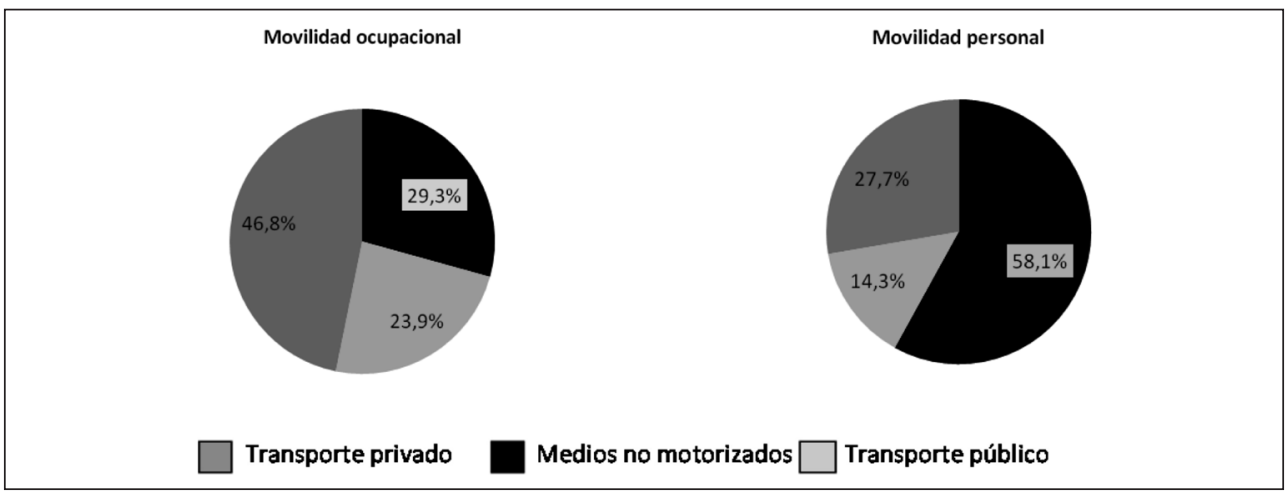

Fuente: ATM PTOP, EMQ 2006. 


\section{COSTES SOCIALES Y MEDIOAMBIENTALES DE LA MOVILIDAD EN LA REGIÓN METROPO- LITANA DE BARCELONA}

El modelo urbano y de movilidad de la región metropolitana de Barcelona no estaría completo si no se añadieran los costes que de ello se derivan. Aquí destacan los sociales y los medioambientales. En los primeros se incluyen todos aquellos aspectos que tienen impactos sobre la sociedad, como los accidentes de tráfico, la expulsión de los usos y de los usuarios del espacio público, la pérdida de autonomía de desplazamiento de la población infantil y el tiempo utilizado, entre muchos otros (EEA, 2001). En los impactos medioambientales uno de sus máximos exponentes es el actual cambio climático, aunque hay otros como la contaminación acústica, la ocupación del suelo o la fragmentación del territorio, etc. (Whitelegg, 1997; Vivier, 2001).

\section{Los costes sociales: el tiempo de desplazamiento y los accidentes de tráfico}

Dentro de los costes sociales vinculados a la movilidad y, consecuentemente, al territorio, sobresalen los relacionados con el tiempo de desplazamiento y con los accidentes de tráfico.

Distintos análisis han evaluado el tiempo total que los barceloneses destinan diariamente a los desplazamientos, los resultados obtenidos en todos ellos son bastante similares, ya que este tiempo se sitúa entre los 80 y los 95 minutos. Valores similares a los que tienen los ciudadanos de otros países europeos ${ }^{8}$, superiores en hombres y en la población más joven. Sin embargo, son los motivos que generan los movimientos, los medios de transporte utilizados y las características de la población los que modulan los tiempos de desplazamiento. Asimismo, los espacios metropolitanos donde discurren tienen también un papel fundamental, perfilándose claramente el centro y la periferia metropolitana: la ciudad de Barcelona y sus distintas coronas.

Según la Encuesta de Movilidad Cotidiana 2006 (EMQ06), en los días laborables, los movimientos por motivos ocupacionales (generados por trabajo y estudio) tienen una duración media de 22,03 minutos por recorrido, y los personales (compras, ocio, etc.), 19,41 minutos. Y es en Barcelona donde los desplazamientos tienen unos valores de tiempo superiores a los del resto de las coronas metropolitanas: en Barcelona superan los 23 minutos, frente a los 20 en la segunda corona. En los motivos personales los valores van de los 21,2 minutos en la ciudad de Barcelona a los 17,9 en la segunda corona. Si la valoración incluye sólo ir al trabajo, el tiempo de recorrido es aún mayor, situándose en casi 26 minutos en Barcelona y en casi 22 en la segunda corona.

La elección del medio de transporte establece la duración temporal media del trayecto. Así, de los tres modos de transporte en que este se divide, son los no motorizados los que presentan un valor temporal menor, con unos 15 minutos de media, los transportes privados tienen unos valores medios de unos 21 minutos y los transportes públicos superan los 37 minutos. A nivel territorial, el transporte que tiene más variabilidad es el público que, a medida que nos alejamos de la ciudad de Barcelona, aumenta sus valores temporales, cuestión relacionada con la oferta de transporte, mucho más amplia - en servicios, frecuencia e

\footnotetext{
8 Eurostat serie Stadistics in focus, transport.
} 
intermodalidad - en el centro metropolitano, especialmente en Barcelona, que en las periferias.

Los modelos de movilidad basados en el uso del transporte motorizado privado implican también elevados costes en forma de accidentes de tráfico, lo que constituye uno de los principales problemas de salud pública en las sociedades occidentales.

En la RMB, tal como indica la tabla 3, la evolución de los accidentes con víctimas y el número de estas ha ido disminuyendo, especialmente en lo que se refiere a la mortalidad en las vías públicas. Disminución que es mayor en las carreteras interurbanas que en las calles de las ciudades, debido a políticas más eficientes y, a la vez, más punibles con las actitudes de riesgo que incrementan la accidentabilidad. El Plan de Movilidad (PDM) de la RMB prevé llegar a una disminución del $25 \%$ de las víctimas mortales en las carreteras entre 2006 y 2012.

Tabla 3

DATOS DE ACCIDENTES SEGÚN ÁMBITO Y CONSECUENCIAS DEL ACCIDENTE 2006-2008

\begin{tabular}{|l|c|c|c|c|c|c|c|c|}
\hline & \multicolumn{4}{|c|}{ Accidentes con víctimas } & \multicolumn{4}{c|}{ Accidentes mortales } \\
\hline \multicolumn{1}{|c|}{ Año } & $\mathbf{2 0 0 6}$ & $\mathbf{2 0 0 7}$ & $\mathbf{2 0 0 8}$ & $\begin{array}{c}\mathbf{\%} \text { Evolución } \\
\mathbf{2 0 0 6 - 2 0 0 8}\end{array}$ & $\mathbf{2 0 0 6}$ & $\mathbf{2 0 0 7}$ & $\mathbf{2 0 0 8}$ & $\begin{array}{c}\text { \% Evolución } \\
\mathbf{2 0 0 6 - 2 0 0 8}\end{array}$ \\
\hline Interurbano & 3.165 & 3.497 & 3353 & $5,94 \%$ & 89 & 65 & 73 & $-17,98 \%$ \\
\hline Urbano & 13.796 & 14.246 & 13.592 & $-1,48 \%$ & 82 & 74 & 61 & $-25,61 \%$ \\
\hline TOTAL & 16.961 & 17.743 & 16.945 & $-0,09 \%$ & 171 & 139 & 134 & $-21,64 \%$ \\
\hline
\end{tabular}

Fuente: Elaboración propia a partir del Anuari estadístic d'accidents a Catalunya 2006, 2007 y 2008, Generalitat de Cataluña.

\section{Costes ambientales: consumo energético, $\mathrm{CO}_{2}, \mathrm{NOx}$}

El transporte es uno de los grandes consumidores de energía final, superando en los últimos años a otros sectores como el doméstico o la industria. Además, es una de las principales fuentes de emisión de gases de efecto invernadero, inductores del cambio climático (Folch, 2008). Las emisiones del transporte doméstico representan el $21 \%$ del total de emisiones de gases de efecto invernadero en la UE y más del $90 \%$ de estas se deben a los transportes por carretera. Entre 1990 y 2005, las emisiones de estos gases en la UE de los 15 se incrementaron un 26\%.

Pero, además, el consumo energético no es homogéneo para todos los medios de transporte mecánicos. Los transportes privados motorizados se sitúan en torno a los 4,5 millones de joules por persona y km recorrido, frente a los 2,8 del autobús y el tren y los 0,8 de la bicicleta (UITP, 2005). Es también el transporte privado el que emite más gases a la atmósfera.

Estas diferencias entre los distintos medios de transporte, asociadas a los costes medioambientales, hacen que los distintos modelos urbanos y de movilidad tengan disfunciones medioambientales distintas. Estas desigualdades distinguen también el centro y las periferias metropolitanas.

En el año 2006, en la RMB, tal como demuestra la tabla 4, para los desplazamientos antes mencionados en transporte privado se utilizaban 3.081.741 vehículos, lo que significaba el 
$63 \%$ de toda Cataluña, con un ratio de 0,64 vehículos por habitante. Un parque automovilístico que utilizaba 2.299.366 Ktep, el 57\% de la energía consumida en Cataluña. Las emisiones de $\mathrm{CO}_{2}$ eran de 6.935.075 t, el 57\% del total catalán, y las de NOx eran de 38.027 t, el $56 \%$ del total catalán.

Tabla 4

COMPARATIVA RMB CATALUÑA 2006

\begin{tabular}{|l|c|c|c|}
\hline & Cataluña & RMB & $\begin{array}{c}\text { Representatividad de la } \\
\text { RMB sobre Cataluña }\end{array}$ \\
\hline Parque de vehículos por combustible & 4.853 .575 & 3.081 .741 & $63,49 \%$ \\
\hline Consumo de energía por tipo de fuente & 4.052 .472 & 2.299 .366 & $56,74 \%$ \\
\hline Emisiones de NOx por tipo de fuente & 68.216 & 38.027 & $55,74 \%$ \\
\hline Emisiones de PM por tipo de fuente & 4.857 & 2.717 & $55,94 \%$ \\
\hline
\end{tabular}

Fuente: Institut Cerdà (2009).

Con el incremento del uso de los transportes privados en los desplazamientos cotidianos y la ampliación de las distancias recorridas, el consumo energético del transporte ha aumentado en los últimos años. Además, el Plan de Movilidad (PDM) de la RMB (2005) prevé un incremento del consumo energético en combustibles fósiles para el transporte entre 2004 y 2012 de un $10,48 \%$, lo que significa un 1,2\% anual. Este mismo plan propone, para el período 2004-2012, una reducción del consumo energético por habitante del 5,8\%.

El PDM de la RMB propone una reducción del 20\% de las emisiones de $\mathrm{CO}_{2}$ entre 2006 y 2012, lo que significa un volumen de emisiones de 12,6 millones de toneladas al final de este periodo. Este mismo documento prevé una reducción del 34,9\% en el año 2012 respecto a 2004, lo que supone una disminución del 5,22\% anual, aunque este plan quiere llegar a unos índices de reducción del 6,1\% anual, lo que supondría entre 2004 y 2012 un total de $39,54 \%$ de disminución. Todo ello con la condición de incrementar los desplazamientos en modos públicos y no motorizados y racionalizar el uso de vehículos privados.

\section{CONCLUSIONES}

Los fenómenos urbanos en el mundo actual son plenamente metropolitanos. La ciudad ya no se desarrolla como un ente territorial aislado de otros núcleos urbanos de su alrededor, tampoco su análisis puede incurrir en aislamientos superados. Al contrario, el fenómeno urbano en el mundo global tiene en las aéreas metropolitanas su exponente territorial.

La ciudad de Barcelona y su entorno metropolitano no es ajena a esta dinámica global. En los últimos años la región metropolitana de Barcelona se ha consolidado a través de dos dinámicas que parecen contradictorias pero que son complementarias. Por un lado, la dispersión y expulsión de ciertas actividades hacia las periferias, por el otro, la recentralización y la concentración de funciones en ciertos barrios de la ciudad capital.

Una y otra dinámica requieren de modelos urbanos distintos, en algunos casos opuestos, que se aprecian a través de las diferencias en las densidades, en las tipologías de vivienda 
y en las distintas pautas que tienen los desplazamientos cotidianos. No es sólo una redistribución de la población en un ámbito metropolitano con un aumento poblacional en las periferias, ni tampoco un incremento de las actividades económicas en la parte más externa de la metrópolis, la segunda corona metropolitana, eso sólo serían las conclusiones lineales de la descripción de unos simples datos. Al contrario, el interés del análisis que aquí se plantea radica en entresacar la complementariedad de estas dos dinámicas y sus consecuencias territoriales.

Un centro más compacto, con una tipología de vivienda más densa y con un uso más intensivo de los modos de transporte menos contaminantes, da lugar a un espacio urbano que alimenta la concentración de ciertas actividades de alto valor añadido. $\mathrm{O}$ al revés, y también cierto, algunas actividades económicas requieren de ciertas características urbanas para desarrollar todo su potencial.

A la vez y en paralelo, las periferias metropolitanas han aumentado sus protagonismos a medida que incrementaban en población y en determinadas actividades urbanas. Estos protagonismos periféricos requieren de otras tipologías territoriales, donde las viviendas unifamiliares, las bajas densidades y la utilización masiva del vehículo privado son sus características fundamentales.

Uno y otro espacio generan costes sociales y ambientales como consecuencias intrínsecas de ciertos modelos territoriales, por lo que no se pueden analizar de forma aislada de las coordenadas espaciales donde se generan. Y además tienen que formar parte de las variables que explican las dinámicas territoriales.

Las dinámicas metropolitanas en la región metropolitana de Barcelona no sólo son una expansión de ciertas actividades, sino también la redefinición del centro y de su periferia. Una redefinición que requiere un análisis multivariable donde la población, las actividades económicas, las tipologías de vivienda, los modelos de movilidad y los costes sociales y medioambientales puedan ayudar a definirlas desde un análisis entre ellas complementario.

Tal como indica Méndez (2009), las periferias han alcanzado un gran protagonismo en los espacios metropolitanos, por ello es en estos territorios donde se juegan el futuro. La consolidación y la estructura de la región metropolitana de Barcelona dependen de cómo se relacione y dialogue un centro muy consolidado y potente, como es la ciudad de Barcelona, y el resto de sus periferias metropolitanas. Un diálogo que requiere de estructuras administrativas específicas, de planeamientos territoriales y de gestión de servicios y equipamientos supramunicipales.

\section{BIBLIOGRAFÍA}

AMIN, S. (1999): Los desafios de la mundialización. México. Siglo XXI.

ASCHER, F. (2004): Los nuevos principios del urbanismo: el fin de las ciudades no está a la orden del día. Madrid: Alianza.

AUTORITAT DE TRANSPORT METROPOLITÀ (ATM) y AJUNTAMENT DE BARCELONA (2004-2008): Enquesta dia feiner (EMEF) 2008. Barcelona. ATM.

AUTORITAT DE TRANSPORT METROPOLITÀ (ATM) y GENERALITAT DE CATALUNYA (2006): Enquesta Mobilitat Quotidiana de Catalunya 2006. Barcelona. ATM. 
BELLET SANFELIU, C. CASELLAS, A. ALONSO, P. (2010): «Infraestructuras de transporte y territorio: los efectos estructurantes de la llegada del tren de alta velocidad en España» en Boletin AGE, nº 52, págs. 143-163.

BELIL, M. (2003): «La ordenación de las aéreas metropolitanas europeas». Planeamiento urbanístico. De la controversia a la renovación (Font, A. coord.). Barcelona, Diputació de Barcelona, 21-37.

BERRY, B. (1980): «Urbanization and Counterurbanization in the United States» en Annals of the American Academy of Political and Social Science, Changing Cities: A Challenge to Planning, núm. 451, 13-20.

BRENNER, N. (2006): «La política de localización el redimensionamiento del Estado y el nuevo gobierno metropolitano en Europa Occidental» en Una nueva cultura del territorio. Criterios sociales y ambientales en las políticas u el gobierno del territorio. CUIMPB, Diputació de Barcelona, 409-430.

CAPEL, H. (2005): La morfología de las ciudades. II aides facere: técnica, cultura y clase social en la construcción de edificios. Barcelona. Ediciones del Serbal.

CAMAGNI, R., GIBELLI, M.C. y RIGAMONTI, P. (2002): «Urban mobility and urban form: the social and environmental costs of different patterns of urban expansion» en Ecological Economics, núm. 40, 191-216.

CARAVACA, I. y FERIA, J.M. (1994): «Universidad y ciudad: necesidades de un nuevo modelo espacial para la universidad de Sevilla» en Annales de geografia de la Universidad Complutense, núm 14. 12 de junio de 2009, 105-118. Universidad Complutense Disponible en http://www.ub.es/geocrit/sn/sn-290.htm

CARAVACA, I y MÉNDEZ, R (2003): «Trayectorias industriales metropolitanas: nuevos procesos, nuevos contrastes» en EURE, vol. XXIX, núm. 87, 37-50.

CUADRADO ROURA, J. R. y DEL RÍO, C. G. (1993): Los Servicios en España. Ediciones Pirámide, Madrid.

CARRERA, J.M. (2002): «Aproximacions a l'estructura espacial de l'àrea metropolitana de Barcelona. diferents maneres d'ocupar el territori» en Papers. Regió Metropolitana de Barcelona, núm. 36, 9-24.

CEBOLLADA, À. (2006): «Aproximación a los procesos de exclusión social a partir de la relación entre territorio y movilidad cotidiana» Documents d'Analisi Geogràfica (DAG), núm. 48, 105-121.

CREVOISIER, O. y CAMAGNI, R. (eds.) (2002): Les milieus urbains: innovation, systèmes de productions et ancrage. Neuchâtel. EDES.

EEA (2001): Term 2001. Indicators Trucking transport and environment integration in the European Union. Copenhagen. European Environment Agency.

FERIA, J.M. (2004): «Problemas de definición de las áreas metropolitanas» Boletín de la AGE núm. 38, 85-99.

FERRAO, J. (2004): «Las regiones metropolitanas como comunidades imaginadas: vivencias, discursos y acción» en Ciudad y Territorio Estudios Territoriales, Vol. XXXVI, núm. 141-142, 517-522.

FILIOL, B., BUNTING, T. y WARRINER, K. (1999): «The entrenchment of urban dispersion: residential preferences and location patters in the dispersed». Urban Studies, núm. 36(8), 1317-1347. 
FLORIDA, R. (2008): Creatives Cities. New York. Basic Book.

FOLCH, R. (2008): «Energia i sostenibilitat. Catalunya 2030» Aula d'Ecologia 2007. Cicle de Conferències. Ciència i Tècnica (Terradas Serra, J. coord.). Bellaterra. Universitat Autónoma Barcelona, 49-56.

GARCÍA, J.C. (2001): «Impacto de las autopista de circunvalación en la accesibilidad del área metropolitana de Madrid» en Estudios Geográficos, núm. 243. 257-283.

GARCÍA, J. (2008): Los desplazamientos al trabajo en la comunidad de Madrid. Madrid, Fundación Sindical de Estudios.

GENERALITAT DE CATALUNYA y SERVEI CATALÀ DE TRÀNSIT (2007-2008-2009): Anuari estadístic d'accidents a Catalunya 2006, 2007, 2008. Barcelona. Generalitat de Catalunya Servei Català de Trànsit.

GENERALITAT DE CATALUNYA. Dpto. PTOP, (2010) Plan Metropolitano de Barcelona PTMB (2010): Pla territorial metropolitá de Barcelona. Barcelona. DOGC n 5627 a $12 / 05 / 2010$.

GIULANO, G. y, SMALL, K.A. (1999): «The determinants of growth of employments subcenters» en Journal of transport Geography, núm. 7, 189-201.

GUTIÉRREZ, J y GARCÍA-PALOMARES J.C (2005): «Cambios en la movilidad en el área metropolitana de Madrid: el creciente uso del transporte privado» en Anales de Geografía de la Universidad Complutense de Madrid, núm. 25, 331-351.

GUTIÉRREZ, J. y GARCÍA-PALOMARES J.C. (2007): «New spatial patterns of mobility within the metropolitan area of Madrid: Towards more complex and disperse flow networks» Journal of Transport Geography, núm. 15(1), 18-30.

HALL, P. y HAY, D. (1980): Growth Centres in the European Urban System. London. Heinemann.

IERMB y IDESCAT (2006): Enquesta de condicions de vida i hàbits de la població de Catalunya. Barcelona, IERMB y IDESCAT.

INSTITUT CERDÀ (2009): Emissions de gasos efecte hivernacle i de contaminants de la mobilitat a Catalunya. Seguiment de les emissions 2004-2006. Barcelona. Generalitat de Catalunya. DPTOP y Autoritat Transport Metropolità.

LOVERING, J. (1999): Theory led by policy: the entrepeneurial City, Chichester, Wiley.

MACLEOD, G. (2001): «New regionalism reconsidered: globalization, regulation and the recasting of political economic space» International Journal of urban and regional Research, núm. 25, 804-829.

MANCOMUNITAT DE MUNICIPIS DE L'AREA METROPOLITANA DE BARCELONA (MMAM) (2003): El territori Metropolità de Barcelona. Dades bàsiques, evolució recent i perspectives. Barcelona.

MARMOLEJO DUARTE, C. y ROCA CLADERA, J. (2008): «La localización intrametropolitana de las actividades de la información: un análisis para la Región Metropolitana de Barcelona 1991-2001» en Scripta Nova, vol. XII, núm. 268, Disponible en http://www. ub.es/geocrit/sn/sn-290 .htm

MELLA, J.M. y LÓPEZ, A. (2006): «Forma urbana y movilidad sostenible: el caso de Madrid» en Revista del Instituto Estudios Económicos, núm. 1-2. 158-189.

MÉNDEZ, R. (2007) «El territorio de las nuevas economias metropolitanas», en Revista EURE, vol. XXXIII, 100. 
MÉNDEZ, R. (2009): «Procesos recientes en regiones metropolitanas. Transformaciones económicas y reorganización territorial. Algunas interpretaciones y debates». La perspectiva geográfica ante los retos de la sociedad y el medioambiente en el contexto Ibérico. Ponéncias del XI Coloquio Ibérico de Geografía. Alcalá de Henares-Pastrana, octubre 2008. Madrid, UAH.

MIRALLES I GUASCH C. y DONAT, C. (2007): «Análisi de l'oferta i la demanda de polígons d'activitat a Catalunya». Papers: Regió Metropolitana de Barcelona, núm. 45, 9-36.

MIRALLES-GUASCH, C. (1998): «La regione metropolitana di Barcellona: tra policentrismo e centralità Economia Púbblica.(Milano e le aree metropolitane in Europa: un confronto) ». Franco Angeli. Anno XXX. Suplemento al nº 3-2000, 81-96.

MIRALLES-GUASCH, C. y CEBOLLADA, A. (2003): Movilidad y transporte. Opciones políticas para la ciudad. Madrid. Fundación Alternativas.

MIRALLES-GUASCH, C. (2002): Ciudad y transporte. El binomio imperfecto. Barcelona. Ariel.

MUÑIZ, I. y GARCÍA-LÓPEZ, M. A. (2010): «The polycentric Knowledge Economy in Barcelona» Urban Geography vol. 31. Núm 6. 774-779.

MUÑOZ, F. (2005): «La producció residencial de baixa densitat» Elements de Debat Territorial núm 21. Barcelona. Diputació de Barcelona.

MUÑOZ, F. (2006): Urbanalització. La producció residencial de baixa densitat a la província de Barcelona 1985-2001. Tesis de doctorado. Bellaterra. Universitat Autònoma Barcelona.

MUÑOZ, F. (2008): Urbanalización. Paisajes comunes, lugares globales. Barcelona. GG Mixta.

NEL.LO, O (2001). Ciutat de ciutats. Reflexió sobre el proces d'urbanització a Catalunya, Editorial Empuries, Barcelona.

ORFUEIL, J.P. y SALOMON, I. (1993): «Travel patterns of the Europeans in every day life» en SALOMON, I. BOVY P. ORFEUIL, J.P. (Eds.) A Billion Trips a Day: Tradition and Transition in European Travel Patterns. Kluver Academic Publishers. Dordrecht, 33-50.

OYON, J. (1999): «Transporte público y estructura urbana: (de mediados s.XIX a mediados s. XX): Gran Bretaña, Francia y países germánicos». Ecología Política. núm. 17, 17-35.

PUJADAS RÚBIES, I. (2009): «Movilidad residencial y expansión urbana en la Región Metropolitana residencial y expansión urbana en la Región Metropolitana de Barcelona, 1982-2005» en Scripta Nova, vol. XIII, núm. 290, 12 de junio de 2009. Universidad de Barcelona. Disponible en http:/www.ub.es/geocrit/sn/sn-290.htm

PRIEMUS, H. NIJKAMP, P. BANISTER, D (2001) Mobility and spatial dynamics: an uneasy relationship Volume 9, Issue 3, September 2001, 167-171

SALOM, J. (2010): «El territorio, escalas y estrategias ». en Territorio, paisaje y sostenibilidad. Un mundo cambiante» (Pillet, F. Cañizares, MC y Ruíz, A.). Barcelona, Ediciones Serbal, 113-136.

RODRIGUEZ, D. A. and JOO, J. (2004): «The Relationship between Non-motorized Mode Choice and the Local Physical Environment» en Transportation Research D, núm. 9(2), 151-173.

SASSEN, S. (2003) «Localizando ciudades en circuitos globales» EURE, Vol. XXIX, núm 88. 5-27. 
STOVER, V.G. and KOEPKE, F.J. (2002): Transportation and Land Development: 2nd ed., Washington, DC, Institute of Transportation Engineers,

TRULLÉN, J., LLADÓS, J. y BOIX, R. (2002): «Economía del conocimiento, ciudad y competitividad». Investigaciones Regionales, núm. 7, 139-163

TRULLEN, J. (2003): «Redes de ciudades y economía del conocimiento. La Cataluña-ciudades». Planeamiento urbanístico. De la controversia a la renovación (Font, A. coord.). Barcelona. Diputació de Barcelona, 21-37.

VALENZUELA RUBIO, M. y SALOM CARRASCO, J. (2008): «Los procesos de urbanización en España: Nuevos factores, nuevas tendencias». España y el Mediterráneo: una reflexión desde la geografía española: Aportación Española al XXXI Congreso de la Unión Geográfica Internacional (Alario Trigueros, M. coord.), Madrid, Ministerio de Fomento, Instituto Geográfico Nacional, 49-56.

VAN DER BERG, L. (1982): Urban Europe: A Study of Growth and Decline. Oxford. Pergamon Press.

VIVIER, J. (2001): The millennium cities Database for sustainable Transport. Brussels. Union International des transports Publics (UITP).

WHITELEGG, J. (1997): Critical mass: transport, environment and society in the twenty first century. London. Pluto. 